\title{
Les principaux types de sapinières (Abies alba Mill) dans le massif du Jura (France et Suisse). Étude phytoécologique
}

\author{
GD Bert \\ INRA, laboratoire de phytoécologie forestière, centre de recherches de Nancy, \\ 54280 Champenoux, France
}

(Reçu le 18 octobre 1991; accepté le 3 décembre 1991)

\begin{abstract}
Résumé - Les premiers résultats d'une étude dendrochronologique ont montré que les peuplements de sapin (Abies alba Mill) dans le Jura ont traversé une période de crise entre 1973 et 1982, due en grande partie à des sécheresses prononcées. Considérées dans leur ensemble, ces sapinières bénéficient depuis d'un net rétablissement de leur croissance radiale. Cependant, certaines d'entre elles sont aujourd'hui encore dépérissantes. L'hypothèse d'une plus grande sensibilité de certaines stations vis-à-vis de stress climatiques a impliqué l'étude de l'interaction entre manifestations du dépérissement et conditions écologiques locales, préalablement mises en évidence pour chaque type de station. L'échantillonnage de 208 sites de sapinières a permis de réaliser une typologie des principales unités stationnelles sur toute l'aire de répartition du sapin dans le Jura. Cet article présente les caractéristiques écologiques et floristiques des 11 principales unités rencontrées au sein de 6 associations phyto-sociologiques : hêtraie à laîche, hêtraie à tilleul, hêtraie à dentaire, hêtraie-sapinière, hêtraie à érable et pessière à doradille. La composition floristique des peuplements est essentiellement conditionnée par l'altitude et le bilan hydrique du site. Les influences climatiques, la biogéographie de certaines espèces et la dissymétrie de la chaîne montagneuse expliquent la disjonction géographique entre certaines unités stationnelles. Certains types de stations floristiquement proches présentent d'importantes différences du point de vue de l'état sanitaire des sapins, apprécié par leur degré de défoliation et de jaunissement. Cette composante stationnelle du dépérissement sera précisée par une étude dendroécologique de la croissance radiale des sapins.
\end{abstract}

typologie des stations / phytoécologie / sapinière / Abies alba = sapin / Jura

Summary - Main types of silver fir (Abies alba Mill) stands in the Jura mountains (France and Switzerland). A phytoecological study. The initial results of a dendrochronological study showed that fir stands underwent a crisis period from 1973 to 1982, mainly due to severe droughts. These stands, considered as a whole, have recovered a normal radial growth level. Nevertheless, some of them are still declining, which suggests that some sites are more sensitive than others to climatic stress. In order to study the relationships between ecological characteristics of sites and dieback symptoms, we determined a typology for silver fir stands. Two hundred and eight sample plots were selected that were as representative as possible of the natural range of silver fir in the Jura mountains (eastern France). This paper describes 11 main types of sites belonging to 6 phytosociologic associations: Carici-Fagetum, Tilio-Fagetum, Dentario-Fagetum, Abieti-Fagetum, AceriFagetum and Asplenio-Piceetum. The ground vegetation depends mainly upon altitude, soil water supply, but also on geographic location due to the distribution of some species and site characteris- 
tics. The soils of the Jura, which is a calcareous massif, consist of rendzina, humic cambisol, calcic cambisol, chromic cambisol, orthic luvisol and chromic luvisols (FAO). A subsequent dendroecological study will deal with differences in state of health between site types.

site typology / phytoecology / fir stand / Abies alba = silver fir / Jura mountains

\section{INTRODUCTION}

Le sapin pectiné, essence indigène du Jura, de grande importance économique, montre des symptômes de dépérissement dans cette région. Pour rechercher les causes de ce dépérissement et en comprendre le mécanisme, des recherches écologiques et dendrochronologiques ont été entreprises dans le contexte phytogéographique jurassien, aux sols calcaires, calciques ou acides. Ces travaux ont permis la comparaison avec les études sur le dépérissement du sapin dans les Vosges, sur des sols en moyenne très acides. Les premiers résultats d'une étude dendrochronologique ont montré que, comme dans les Vosges (Becker, 1987; Becker et Lévy, 1988), les sapinières jurassiennes, considérées dans leur ensemble, bénéficient d'un net rétablissement du point de vue de la croissance radiale, après une période de crise entre 1973 et 1982 due en grande partie à des sécheresses prononcées (Bert et Becker, 1990).

Cependant, certains peuplements sont aujourd'hui encore dépérissants. Au cours de travaux ultérieurs, nous chercherons donc à tester l'hypothèse d'une plus grande sensibilité de certains arbres ou de certaines stations vis-à-vis de stress climatiques, éventuellement aggravée par de mauvaises conditions sylvicoles ou l'intervention de polluants. L'étude de l'interaction possible entre conditions écologiques locales et manifestation du dépérissement passe par l'utilisation d'une typologie des stations préalablement construite. En effet, la réalisation d'une classification de la végétation met en évidence les principaux facteurs du milieu qui structurent la flore
(Pfister, 1989) et les caractéristiques écologiques de chaque type de station. Cette typologie était encore fragmentaire du point de vue géographique et hétérogène pour une utilisation sur l'ensemble du massif. Moor (1952) a étudié les associations du Fagion dans le Jura suisse, Richard (1961) a traité des forêts acidophiles du Jura suisse et de la moitié nord du jura français, Guinochet (1973) a cartographié très finement une petite région au sud de Pontarlier, Simmeray (1976) a interprété les groupements de la région de Saint-Claude, Gaiffe et Schmitt (1980) ont analysé la végétation de Mouthe à Pontarlier, Gillet et al (1984) ont réalisé une étude cartographique de la région sud-est du massif et Rameau (1988) a décrit les hêtraies mésoneutrophiles et acidiclines de la partie ouest du Jura.

Le dispositif initial de 87 placettes, mis en place en 1987, dans les départements du Doubs et du Jura, a été étendu à l'ensemble du massif, tant dans sa partie française que suisse. Ainsi, notre étude de terrain a permis de rassembler les éléments nécessaires à un examen général de la variabilité stationnelle à l'échelle du massif. L'analyse a conduit dans un premier temps à identifier les associations végétales forestières déjà définies sur le Jura. Puis nous avons précisé la variabilité stationnelle de chacune d'elles afin de définir des unités stationnelles plus homogènes. Ces unités stationnelles ne correspondent pas toujours à des "types de stations" (unités parfaitement homogènes du point de vue stationnel) car l'échantillonnage a été réalisé dans un but dendroécologique.

Cet article présente les résultats phytoécologiques issus de l'analyse de la végéta- 
tion et les caractéristiques écologiques et géographiques des unités stationnelles.

\section{AIRE D'ÉTUDE, MÉTHODES}

\section{Limites géographiques}

La surface étudiée couvre l'aire de répartition du sapin dans le massif du Jura. La limite des sapinières correspond au rebord du second plateau à $650 \mathrm{~m}$ d'altitude. Les précipitations moyennes annuelles dépassent $1200 \mathrm{~mm}$. L'ensemble des 208 peuplements échantillonnés s'étend entre
Delémont (en Suisse, $30 \mathrm{~km}$ au SE de Bâle) et le lac du Bourget $(35 \mathrm{~km}$ au Nord de Chambéry), soit une surface de $230 \times 50 \mathrm{~km}$ (fig 1). La partie française du massif compte 144 sites : 77 dans le département du Doubs, 41 dans le Jura et 26 dans l'Ain; les 64 sites suisses se partagent entre les cantons du Jura (11 placettes), de Berne (13), de Neuchâtel (19) et de Vaud (21).

\section{Échantillonnage, observations phytoécologiques}

L'échantillonnage n'a concerné que les stations sur lesquelles les peuplements forestiers com-

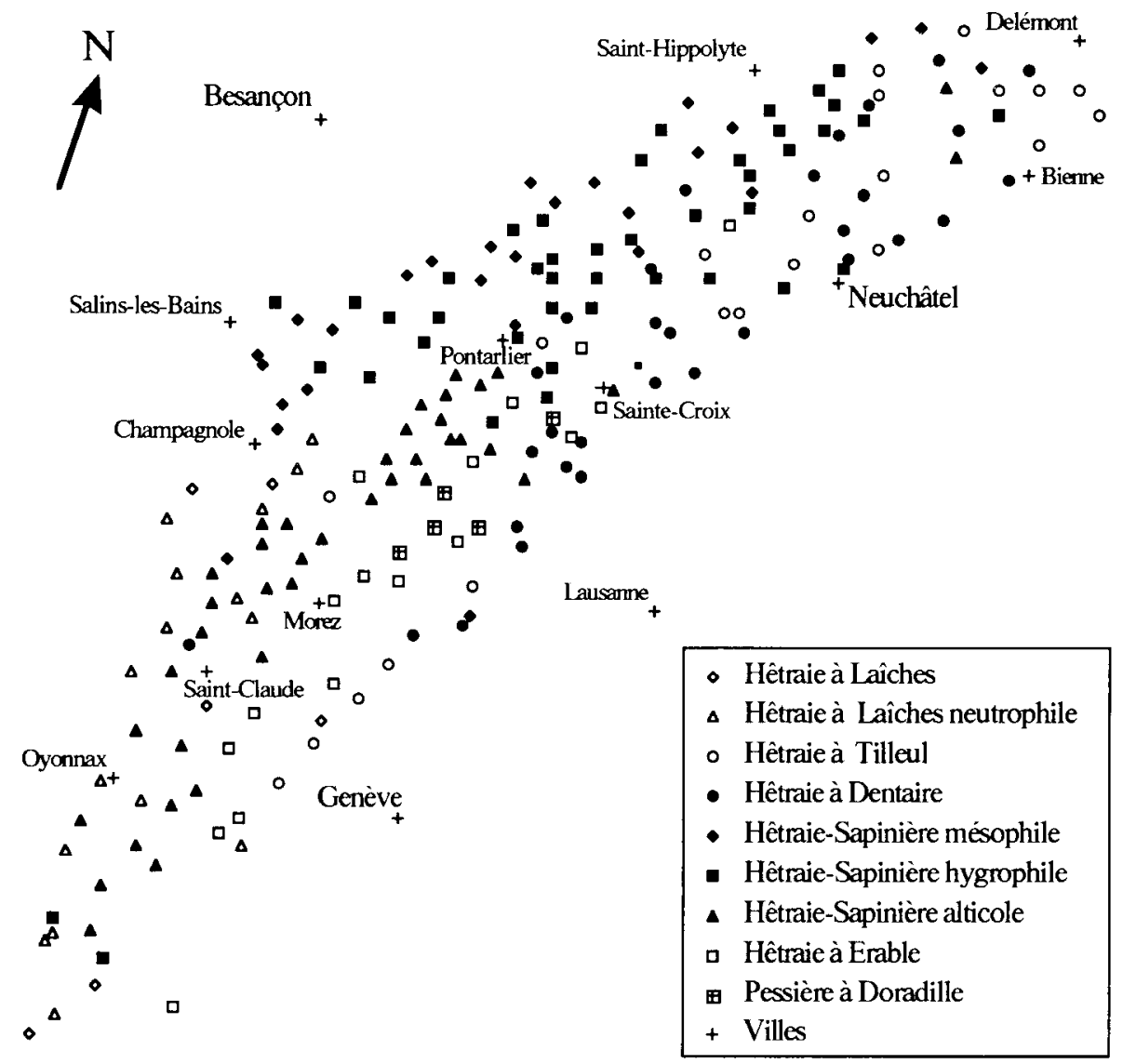

Fig 1. Localisation géographique et type de station des 208 peuplements de sapin (Abies alba) échantillonnés dans le Jura. 
portaient plus de $50 \%$ de sapin; toutes les associations végétales ne pouvaient donc pas être rencontrées. Le choix des placettes a été fait en respectant la variabilité des conditions stationnelles au sein d'une même petite région. Les peuplements de sapins échantillonnés couvrent une large gamme d'âges, afin de pouvoir réaliser une étude dendrochronologique. Chaque placette d'une surface de $400 \mathrm{~m}^{2}$ comportait 6 sapins dominants sur lesquels diverses mesures dendrométriques et observations sanitaires ont été réalisées. La défoliation (pourcentage d'aiguilles absentes par rapport à un sapin sain de même morphologie) et le jaunissement (pourcentage d'aiguilles jaunes dans le houppier) ont été appréciés par le même notateur sur les 1248 sapins. L'état sanitaire des sapins n'a pas conditionné leur choix pour conserver une bonne représentativité de l'échantillon visà-vis du degré de dépérissement de l'ensemble des sapinières.

Un relevé phytoécologique a été réalisé sur chaque placette. La composition floristique a été notée en nommant toutes les espèces selon la nomenclature de Flora Europaea (Halliday et Beadle, 1983) et en notant leur abondancedominance selon la méthode «BraunBlanquet". Le peuplement a été brièvement caractérisé par son type de traitement en "futaie régulière" ou ufutaie jardinée». Le site a été décrit par : les coordonnées géographiques, l'altitude (mesurée avec un altimètre, précision = $10 \mathrm{~m}$ ), la pente (en degrés), l'azimut magnétique du haut de la pente, le masque (pente de la droite joignant la placette au sommet du versant opposé); ces 3 dernières valeurs ont été intégrées en un «indice de climat radiatif" (Becker, 1982) qui varie dans l'échantillon entre 0,25 (stations «froides") à 1,25 (stations "chaudes"). La position topographique est notée comme «sommet", «haut de pente», "mipente", «plateau", "bas de pente" ou "dépression". Le sol a été décrit grâce à une fosse pédologique ou un prélèvement a la tarière, selon sa charge en éléments grossiers; les descripteurs principaux sont : la profondeur de décarbonatation, la profondeur de sol, le type de substrat classé en "marne", "banc calcaire compact", "banc diaclasé", «banc concassé", "éboulis fins", "éboulis grossiers" et «moraines». Ces éléments permettent de rattacher le sol aux types décrits par Gaiffe et Schmitt (1980), Bruckert et Gaiffe (1980) en suivant la nomenclature de la classification des sols CPCS (1967). Le rattachement a été parfois compliqué par la disposition des sols en mosaïque : le type de sol varie sur une courte distance selon la microtopographie et la structure du substrat sous-jacent. De même, l'abondance de cailloux dans les profils ont rendu délicate la mesure de profondeur de sol. Afin d'harmoniser les dénominations, nous avons également exprimé les noms de sols selon la nomenclature du Référentiel pédologique français (Baize et Girard, 1990). La fosse pédologique la plus caractéristique de chaque type de sol a fait l'objet de prélèvements et d'analyses; les résultats de l'analyse granulométrique ont permis de calculer la réserve en eau moyenne.

\section{Méthodes d'analyse}

Les relevés floristiques en abondancedominance ont été soumis aux analyses multivariées : analyse factorielle des correspondances (AFC) et classification ascendante hiérarchique (CAH) du logiciel SPAD N (Lebart et al, 1988).

L'analyse factorielle des correspondances est essentiellement un mode de représentation graphique du tableau de contingence constitué par les 208 relevés et les 199 espèces caractérisées par leur coefficient d'abondance-dominance ( $L \theta-$ bart et al, 1977). Elle utilise 2 types de variables. Les variables actives (ou principales) déterminent les axes; ce sont les 199 espèces de cette étude. Les variables supplémentaires ne participent pas au calcul des valeurs propres mais elles peuvent être représentées sur les plans factoriels comme le barycentre d'un groupe de relevés. Dans le cas de notre étude, les relevés ont été regroupés selon 9 tranches d'altitude, puis le point moyen de chaque groupe a été projeté sur l'AFC. La même procédure a été employée pour les différentes variables stationnelles : type de sol, profondeur de sol, indice de climat radiatif. L'agencement des $n$ points moyens correspondant aux $n$ classes d'une variable au long d'un axe permet de conforter son interprétation écologique.

L'analyse factorielle permet d'établir la correspondance entre le nuage de points des relevés et celui des espèces. Dans un premier temps, l'interprétation de l'agencement des espèces les unes par rapport aux autres révèle les facteurs écologiques essentiels qui structurent la flore. Ceux-ci sont mis en évidence en observant le mode de regroupement des especes, 
dont on connaît le caractère indicateur, qui contribuent le plus à la création d'un axe. Le caractère indicateur de chaque espèce est révélé par l'observation de ses profils écologiques en fonction des différentes variables stationnelles. Les variables supplémentaires confortent l'interprétation des axes réalisée à partir des espèces. Dans un second temps, le regroupement des placettes est interprété à l'aide de ces mêmes facteurs.

Si les relevés ont été faits dans des milieux nettement différents et distants les uns des autres, les groupes de relevés se séparent aisément sur les plans factoriels. Au contraire, si les relevés ont été réalisés au sein de gradients écologiques continus, au long d'un transect par exemple, les plans factoriels à 2 dimensions montrent un nuage de points homogène : les relevés ne sont pas individualisés en groupes. Dans ce cas, il est nécessaire de réaliser une classification automatique des relevés.

La classification ascendante hiérarchique fournit un ensemble de classes de moins en moins fines obtenues par regroupement successif de parties. Le dendrogramme, ou arbre de classification, est obtenu de manière ascendante : on regroupe d'abord les 2 relevés floristiquement les plus "proches" qui forment un "sommet", il ne reste alors plus que $(n-1)$ objets et le processus est réitéré jusqu'à regroupement complet (Saporta, 1990). L'algorithme de SPAD $\mathrm{N}$ fonctionne avec le critère d'agrégation de Ward qui utilise la distance euclidienne. Les calculs peuvent être effectués sur les relevés floristiques, ce qui est objectif mais englobe les variations aléatoires de leur composition, ou sur les premiers axes de l'AFC. Dans ce cas, la classification ne prend en compte que l'information de ces axes et ignore les facteurs non interprétables ou de faible poids. Nous avons effectué la $\mathrm{CAH}$ sur les 10 premiers axes de l'AFC.

La qualité de l'appartenance d'un relevé à un groupe issu de la classification hiérarchique a été chiffrée à l'aide d'une nouvelle méthode : la classification par "ensembles flous". Le module Fuzzy contenu dans le logiciel Modulad (Dupouey, 1989) fonctionne selon ce priricipe. La classification par «ensembles flous» affecte à chaque relevé une valeur d'appartenance (variant entre 0 et 1) à chacun des groupes floristiques. Avant cette analyse, les relevés sont regroupés selon les résultats de la classification hiérarchique; après calculs, les relevés "typiques» de chacun des groupes peuvent être distingués des relevés «hybrides", situés entre les groupes (Dupouey, 1985; Equihua, 1990).

Pour situer notre typologie par rapport aux travaux antérieurs et rattacher ses unités stationnelles à des descriptions antérieures, 36 relevés issu de la littérature ont été inclus dans l'analyse factorielle des correspondances en tant que relevés supplémentaires (Richard, 1961; Simmeray, 1976; Gillet et al, 1984). Ces individus supplémentaires ne peuvent pas modifier l'analyse; leur projection sur le plan formé par les 2 premiers axes de l'AFC est réalisée en prenant pour référence l'ensemble des relevés propres à la présente étude.

\section{RÉSULTATS}

\section{Interprétation de l'analyse factorielle des correspondances}

Les 3 premiers axes expliquent $14,4 \%$ de la variance du nuage de points. Ils ont pu être interprétés en termes de facteurs écologiques qui influent sur la végétation.

\section{Axe $1(6,2 \%$ de la variance du nuage)}

Sur cet axe, les espèces dont le profil écologique indique une préférence pour les faibles altitudes se localisent du côté des plus faibles abscisses: Carpinus betulus, Cornus sanguinea, Crataegus laevigata, C monogyna, Fraxinus exelsior, Ligustrum vulgare, Quercus petraea. Au contraire, les espèces à caractère indicateur montagnard correspondent aux plus fortes abscisses: Adenostyles alpina, Asplenium viride, Lonicera alpigena, Orthilia secunda, Ranunculus aconitifolius, Rubus saxatilis, Vaccinium myrtillus. Par ailleurs, la projection du point moyen des 9 classes d'altitude montre un agencement très net au long de l'axe 1 (fig 2). La corrélation entre la coordonnée sur l'axe 1 d'une placette et l'altitude est significative à $0,1 \%(r=0,80)$. 
Ce premier axe est donc interprété comme un gradient climatique lié à l'altitude du site.

\section{Axe 2 (4,6\% de la variance du nuage)}

Les espèces indicatrices de sols riches en squelette calcaire (sols humo-calcaires, humo-calciques) ou d'une faible décarbonatation possèdent les plus fortes coordonnées sur l'axe 2, et inversement pour les espèces les plus acidiphiles, ce qui semblerait indiquer un gradient trophique. Cependant, l'étude de la répartition des espèces sensibles au régime hydrique du sol a révélé le véritable facteur écologique exprimé par cet axe : un gradient d'humidité, ou de bilan hydrique de la station. Le bilan hydrique a été estimé grâce à la profondeur de sol, la position topographique, la pente de la station ou la perméabilité de la roche mère. Les 2 hypothèses sont cependant compatibles car les sols calcaires sont également les moins épais (30-50 $\mathrm{cm}$ environ) et les plus secs, tandis que les sols bruns plus acides sont épais (60 à plus de $110 \mathrm{~cm}$ ) et présents sur des stations où le drainage est inférieur aux apports d'eau. La projection des points moyens des variables stationnelles met en évidence les mêmes résultats, avec moins d'efficacité (fig 2). D'autres résultats, portant notamment sur l'analyse de la croissance en hauteur des sapins, ont confirmé nettement l'interprétation de l'axe 2 grâce au bilan hydrique (Bert, 1992).

\section{Axe 3 (3,6\% de la variance du nuage)}

Les variables stationnelles ne permettent pas une interprétation satisfaisante de l'axe 3. On a cherché à voir si la position relative des points relevés le long de cet axe ne mettait pas en évidence une structuration d'ordre géographique à laquelle correspondrait un déterminisme écologique. Les placettes dont la coordonnée sur l'axe 3 est négative se localisent géographiquement sur la bordure ouest du Jura; les placettes avec des coordonnées posjtives se localisent à l'est du massif. Par ailleurs, la répartition géographique des espèces au sein du massif permet de les rattacher à 5 principaux types :

- espèces collinéennes sur le pourtour du Jura : Acer campestre, Crataegus laevigata, Ligustrum vulgare, Quercus petraea;

- espèces montagnardes sur la HauteChaîne : Lonicera alpigena, Moehringia muscosa, Ranunculus aconitifolius, R lanuginosus, Valeriana montana, Veronica urticifolia;

- espèces xérophiles au sud : Carex alba, Coronilla emerus, Laburnum anagyroides, Teucrium scorodonia;

- espèces continentales situées préférentiellement au nord-est : Lilium martagon, Maianthemum bifolium, Prunus avium, Sorbus mougeoti, Stachys officinalis;

- espèces mésophiles ou hygroclines, subocéaniques, plus abondantes dans le quart nord-ouest : Carex remota, Impatiens nolitangere, Glechoma hederacea, Senecio fuchsii, Veronica montana...

L'axe 3 révèle donc un gradient de continentalité. Il apparaît au travers du dispositif car le Jura est soumis aux 3 grands types d'influences climatiques et de courants de migrations végétales : méditerranéenne, atlantique et continentale.

\section{Structuration des relevés}

L'AFC a montré que la composition floristique des groupements végétaux dépend principalement de l'action conjuguée de l'altitude, de l'humidité du sol et de leur position géographique. La classification ascendante hiérarchique a débord séparé les relevés d'altitude des stations de basse altitude. Au sein de ces dernières, les sta- 

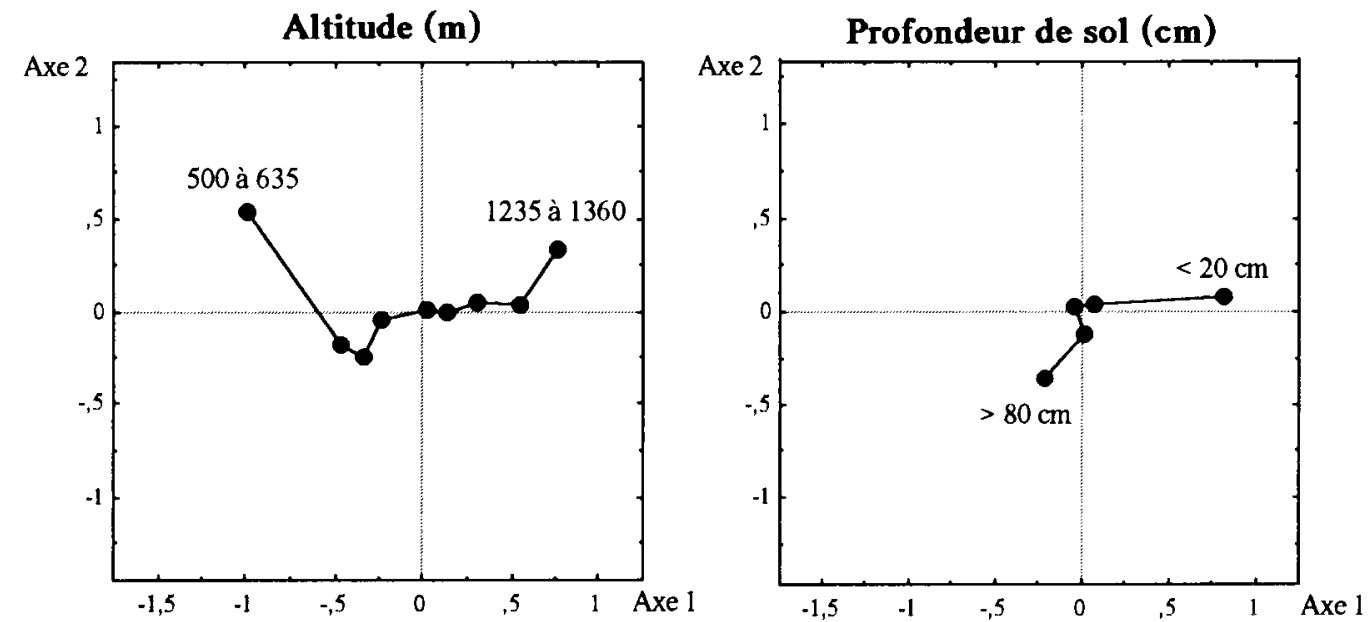

Type de sol
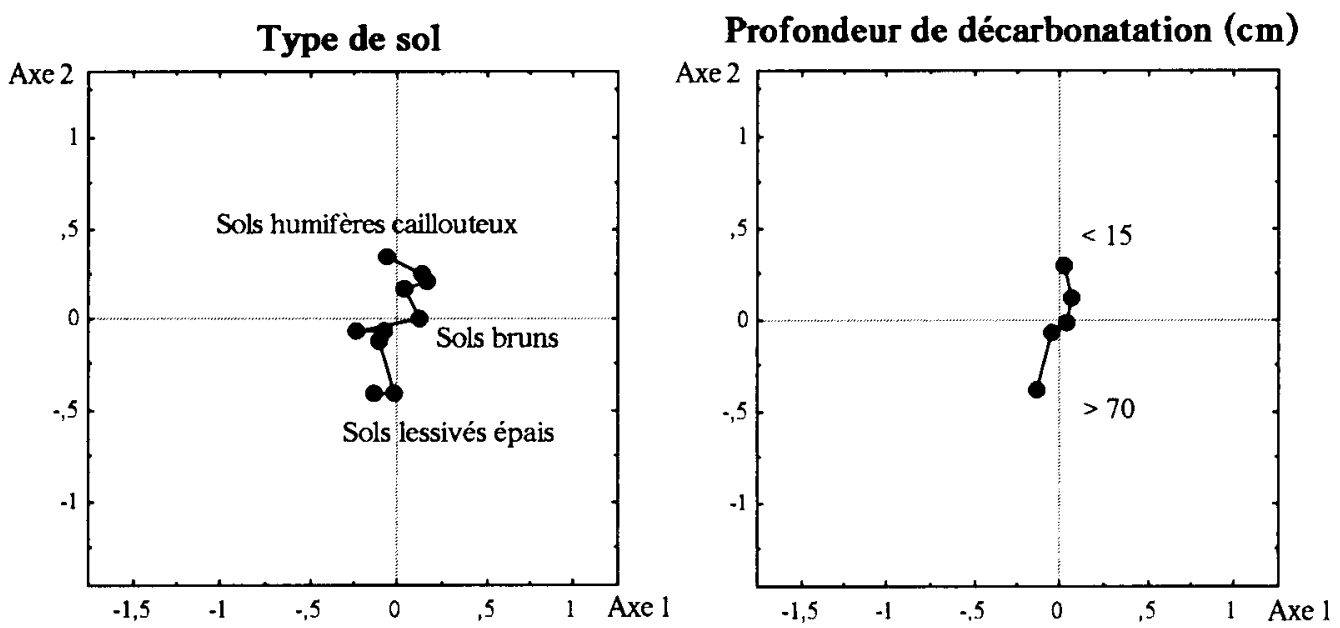

Fig 2. Projection sur le plan formé par les 2 premiers axes de l'AFC des points moyens correspondant aux relevés regroupés par classe d'altitude, de profondeur de sol, de type de sol ou de profondeur de décarbonatation.

tions sèches ont été séparées des stations hygrosciaphiles; enfin, ces dernières ont été subdivisées en hêtraies à dentaire et hêtraies-sapinières (fig 3). Finalement, 11 unités stationnelles ont été identifiées et ont pu être rattachées à des unités décrites par Moor (1968), Gaiffe et Schmitt
(1980), Rameau et al (1980) et Rameau (1987).

La projection des groupes de relevés sur le plan formé par les 2 premiers axes de l'AFC (en 2 dimensions) montre des ensembles assez enchevêtrés. Pour en épurer la représentation, nous avons transfor- 
mé ce plan en un diagramme qui combine les gradients altitudinal et hydrique; il montre l'amplitude de répartition écologique des diverses associations (fig 4).

Les facteurs essentiels qui structurent la végétation ont été utilisés pour présenter les unités stationnelles forestières sous forme d'un tableau floristique (tableau I). Les relevés ont été ordonnés en fonction de l'altitude, exprimée par leur coordonnée sur l'axe 1. Les associations les plus collinéennes sont sur la gauche du tableau et les plus montagnardes sont à l'autre extrémité. Le découpage présenté dans le tableau a été conditionné par la taille de l'échantillon réalisé; les unités pourraient être plus finement décomposées en augmentant le nombre de points de relevés.

Les 4 groupes d'espèces individualisés par une $\mathrm{CAH}$ correspondent globalement aux étages bioclimatiques submontagnard, montagnard inférieur, montagnard moyen, montagnard supérieur/subalpin. Plus précisément, certaines espèces sont effectivement bien liées à une variation d'altitude tandis que le classement d'autres espèces dans un "étage» est dû à l'échantillon de relevés disponibles pour cette étude. Ce gradient a cependant été conservé pour classer les espèces dans le tableau pour mettre en évidence les affinités entre espèces aux diverses altitudes. Au sein de chaque étage, les espèces ont été ordonnées par groupes écologiques, en fonction d'un gradient hydrique (prépondérant) et trophique, comme l'indique la légende au bas du tableau. Les espèces différentielles de chaque groupe sont encadrées.

\section{Associations végétales et unités stationnelles}

La description des différents groupes comporte leurs principales différentielles floris- tiques, écologiques et pédologiques. Les associations végétales sont nommées selon la nomenclature européenne reprise par Gillet et al (1984). Elles sont présentées selon le gradient principal d'altitude croissante. Le tableau II donne le résultat des analyses effectuées sur les 10 sols les plus typiques de l'échantillon.

\section{Hêtraie à laîches}

Cette association appartient au CariciFagetum Moor 1952. Elle s'individualise floristiquement grâce à la présence d'espèces de "faible» altitude (étages submontagnard et montagnard inférieur), calcaricoles, calcicoles, neutrocalcicoles et calciclines : Carex alba, Mercurialis perennis (caractérisent le groupement), Acer campestre, Carex flacca, Clematis vitalba, Cornus sanguinea, Crataegus monogyna, Daphne laureola, Ligustrum vulgare, Lonicera xylosteum, Melica mutans, Sesleria albicans, Teucrium scorodonia. Les placettes qui représentent la hêtraie à laîches s'étagent principalement entre 580 et 950 $m$ d'altitude (fig 5), de préférence sur les versants chauds. Elles sont situées sur des éboulis grossiers, en position de mipente sur des pentes moyennes à fortes $\left(10-25^{\circ}\right)$.

La hêtraie à laîches se rencontre sur des sols humifères carbonatés ou peu décarbonatés : humo-calcaire ou humocalcique. Les sapins de ce milieu montrent un important manque d'aiguilles dans leur houppier, en moyenne $21 \%$, et les plus forts jaunissements du feuillage.

\section{Hêtraie à laîches neutrophile}

Richard (1961) a distingué 2 sousassociations de hêtraie à laîches : le Carici-Fagetum caricetosum albae (Moor, 1952), caractérisé par Carex alba et que 
Pessière à Doradille

Hêtraie à Erable

* mésotrophe

* neutrophile

Hêtraie-Sapinière

* alticole

Sapinière à Prêles

Hêtraie-Sapinière

* hygrophile

* mésophile

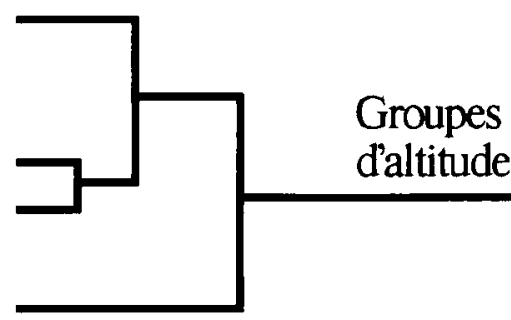

Hêtraie à Dentaire

Hêtraie à T:lleul

Hêtraie à Millet

Hêtraie à Adénostyle

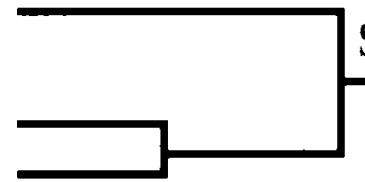

Sapinières

Hêtraie à Laîches neutrophile

Hêtraie à Laîches

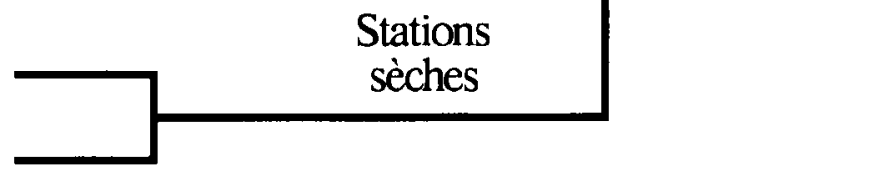

Fig 3. Types de stations mis en évidence par classification ascendante hiérarchique. Le niveau de regroupement sur le dendrogramme indique le degré de ressemblance floristique entre les groupes.

nous avons nommé "hêtraie à laîches", et une sous-association légèrement acidocline de la hêtraie à laîches : le CariciFagetum caricetosum montanae, caractérisé par Carex montana. L'analyse a distingué ce second groupement grâce à la présence d'espèces neutrocalcicoles, calci- clines, neutroclines ou acidiclines : Athyrium filix-femina, Carex sylvatica, Dryopteris dilatata, $D$ filix-mas, Hylocomium splendens, Hordelymus europaeus, Rubus sp, Rhytidiadelphus loreus, Tilia platyphyllos, Thuidium tamariscinum. Cette sousassociation se rencontre à l'étage submon- 
Are 2

Bilan hydrique

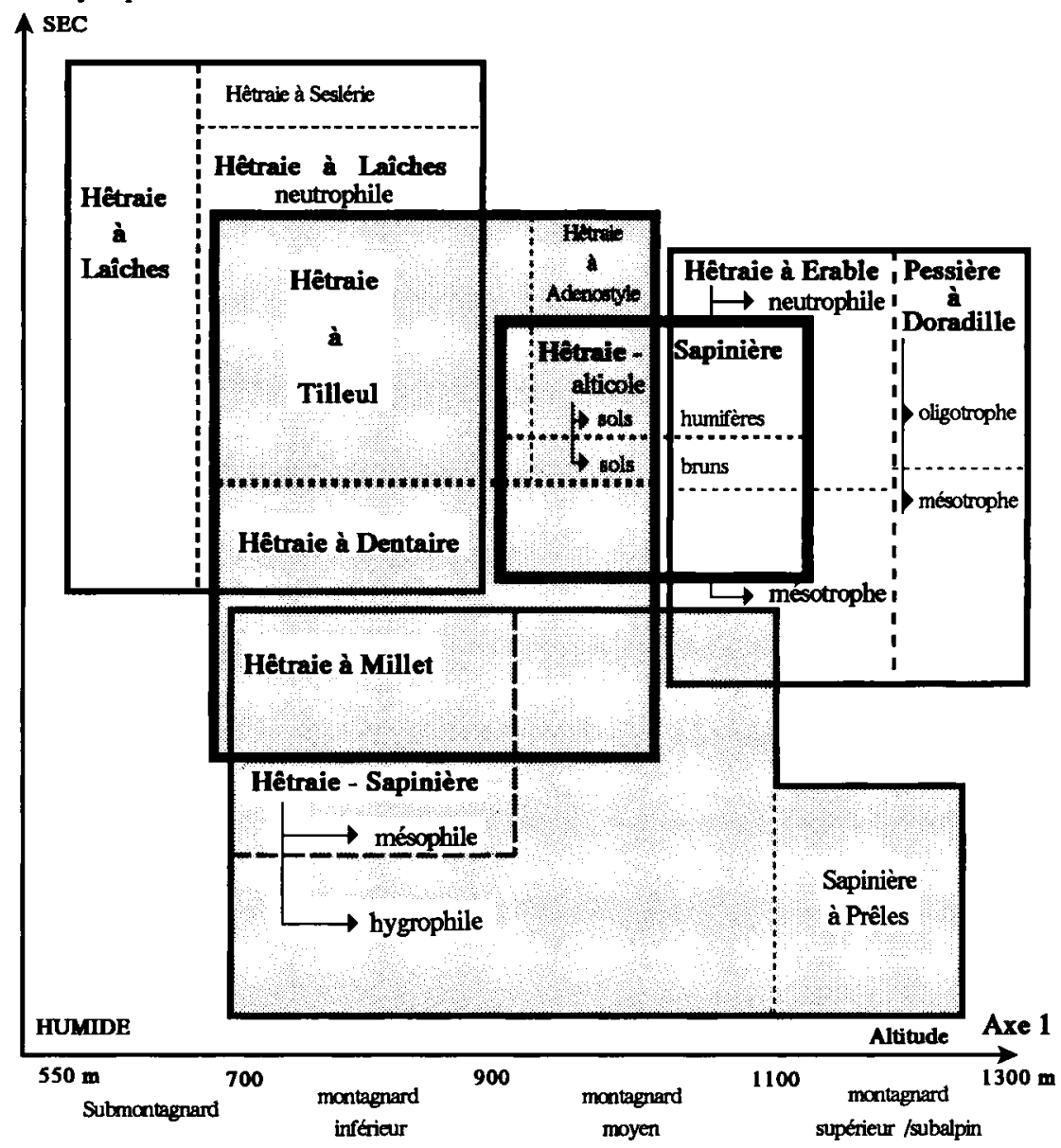

Fig 4. Projection schématisée sur le plan formé par les 2 premiers axes de l'AFC des groupes de relevés floristiques : variabilité écologique de chaque association au long des gradients d'altitude (Axe 1) et d'humidité moyenne du sol (Axe 2). Les groupes floristiques de la moitié nord du massif sont grisés.

tagnard/montagnard inférieur entre 700 et $860 \mathrm{~m}$. Le substrat est constitué par des bancs calcaires diaclasés ou concassés, ou des éboulis grossiers, en positions topographiques variées (srutout en mipente) sur des pentes faibles de $3-16^{\circ}$.
Cette dernière caractéristique explique l'absence de colluvionnement des sols humo-calciques ou humo-calcaires. La perméabilité du substrat confère un caractère relativement xérique aux stations de hêtraie à laîches : précipitations de 1300 à 
$1500 \mathrm{~mm}$, réserve estimée en eau utile de $70 \mathrm{~mm}$. Les sapins dominants sont souvent parasités par du gui (Viscum album) et jaunissants. Les 24 sites qui représentent la hêtraie à laîches n'ont pas permis de distinguer les 15 sous-associations décrites plus récemment par Moor (1972).

\section{Hêtraie à seslérie}

L'association Seslerio-Fagetum a été décrite par Moor (1952); elle est caractérisée par Ses/eria albicans. Elle se rencontre sur les stations les plus sèches, sur des sols carbonatés superficiels. Un seul site la représente dans notre échantillon et nous l'avons regroupé avec ceux de la hêtraie à laîches.

\section{Hêtraie à tilleul}

Le Tilio-Fagetum Moor 1952 se rencontre sur pente forte $\left(12-35^{\circ}\right)$ et peu stabilisée, en position de haut de pente ou de mipente, entre 800 et $1050 \mathrm{~m}$. La flore est marquée par la présence d'espèces calcicoles : Acer campestre, Daphne laureola, Helleborus foetidus, Melittis melissophyllum, Melica nutans, Tilia platyphyllos. L'abondance d'espèces neutroclines et neutronitroclines la distingue de la hêtraie à laîches neutrophile : Acer pseudoplatanus, Euphorbia amygdaloides, Fissidens taxifolius, Lilium martagon, Phyteuma spicatum, Prenanthes purpurea, Solidago virgaurea. Les stations sont exposées au nord-ouest ou au nord-est et plutôt au nord si l'altitude est faible.

Les substrats sont drainants : banc calcaire concassé ou éboulis. Les sols sont le plus souvent du type humo-calcaire colluvial (Calcosol humique caillouteux sur éboulis, selon le Référentiel pédologique) ou humocalcique; ils renferment peu de terre fine entre les éléments du squelette calcaire, ce qui les distingue des sols de la hêtraie à dentaire. Ce type de sol est formé de 2 horizons très caillouteux (plus de $65 \%$ en poids) riches en matière organique, aux $\mathrm{pH}$ élevés $(\mathrm{pH}$ eau $=7,7$ dans I'horizon $A_{1}$ et 8 dans le $A_{1} C$ ); la terre fine fait effervescence sous l'action de l'acide et contient 50 à $100 \mathrm{~g}$ de $\mathrm{CaCO}_{3}$ total par $\mathrm{kg}$. Les sols humo-calcaires ont une épaisseur de 30 à $55 \mathrm{~cm}$ et leur réserve en eau est très faible : environ $60 \mathrm{~mm}$.

\section{Hêtraie à dentaire}

Les stations de hêtraie à dentaire font partie du Dentario-Fagetum Moor, 1952; Th Müll 1966. L'association est caractérisée par Fagus sylvatica, Cardamine heptaphylla et Actaea spicata. Les sols à réserve assez importante expliquent le caractère mésophile de la flore de ces stations : Acer pseudoplatanus, Bromus ramosus, Carex sylvatica, Circaea lutetiana, Fraxinus exelsior, Geranium robertianum, Paris quadrifolia, Prenanthes purpurea, Sambuscus nigra, Sanicula europaea, Plagiomnium undulatum. Ce groupement est très répandu à l'étage montagnard moyen, entre 800 et $1050 \mathrm{~m}$, sur des pentes moyennes de 6-22 . II se rencontre principalement sur des substrats calcaires drainants (banc diaclasé ou concassé, éboulis) qui permettent l'existence de sols bruns calciques (Calcisol argileux, selon le Référentiel pédologique). La flore traduit le caractère calcicole ou neutrocalcicole du milieu : Cardamine heptaphylla, Mercurialis perennis, Mycelis muralis, Fissidens taxifolius. Les précipitations, comprises entre 1400 et $1600 \mathrm{~mm} / \mathrm{an}$, et réparties régulièrement, fournissent l'humidité nécessaire à la croissance du hêtre. La moyenne annuelle des températures oscille entre 7 et $8{ }^{\circ} \mathrm{C}$ (Simmeray, 1976).

Le sol brun calcique est décarbonaté sur la majeure partie de son profil. L'horizon $A_{1}$, de $15 \mathrm{~cm}$, est limoneux-argileux et 
Tableau II. Résultats de l'analyse d'un échantillon des 10 principaux types de sols de sapinières jusols. INRA-Arras).

\begin{tabular}{|c|c|c|c|c|c|c|c|c|}
\hline $\begin{array}{l}\text { Sol } \\
\text { Unité stationnelle associée }\end{array}$ & Horizon & $\begin{array}{l}\text { Profond } \\
\text { (cm) }\end{array}$ & $\begin{array}{l}\text { Elem Gros } \\
\text { (\%) poids }\end{array}$ & $A$ & $\begin{array}{l}L F \\
(\%) p c\end{array}$ & $\begin{array}{l}L G \\
\text { ids terr }\end{array}$ & $\begin{array}{l}S F \\
\text { fine }\end{array}$ & $S G$ \\
\hline $\begin{array}{l}\text { Humocalcaire } \\
\text { Hêtraie à laîches }\end{array}$ & $\begin{array}{r}A_{1} \\
A_{1} C\end{array}$ & $\begin{array}{c}0-20 \\
20-30\end{array}$ & $\begin{array}{l}80 \\
80\end{array}$ & $\begin{array}{l}26,5 \\
22,2\end{array}$ & $\begin{array}{l}42,4 \\
39,9\end{array}$ & $\begin{array}{l}17,4 \\
12,1\end{array}$ & $\begin{array}{l}5,6 \\
5,5\end{array}$ & $\begin{array}{r}8,1 \\
20,3\end{array}$ \\
\hline $\begin{array}{l}\text { Humo-calcaire colluvial } \\
\text { Hêtraie à tilleul }\end{array}$ & $\begin{array}{l}A_{1} \\
A_{1} C\end{array}$ & $\begin{array}{c}0-10 \\
10-40\end{array}$ & $\begin{array}{l}65 \\
70\end{array}$ & $\begin{array}{l}29,1 \\
21,9\end{array}$ & $\begin{array}{l}41,8 \\
38,9\end{array}$ & $\begin{array}{l}17,5 \\
24,8\end{array}$ & $\begin{array}{l}6,3 \\
8\end{array}$ & $\begin{array}{l}5,3 \\
6,4\end{array}$ \\
\hline $\begin{array}{l}\text { Humo-calcique sur banc } \\
\text { Hêtraie-sapinière alticole }\end{array}$ & $\begin{array}{l}A_{1} \\
A_{1} C\end{array}$ & $\begin{array}{c}0-30 \\
30-50\end{array}$ & $\begin{array}{l}95 \\
75\end{array}$ & $\begin{array}{l}29,5 \\
23,9\end{array}$ & $\begin{array}{l}47,6 \\
36,9\end{array}$ & $\begin{array}{l}17,2 \\
25,9\end{array}$ & $\begin{array}{l}3,4 \\
9,2\end{array}$ & $\begin{array}{l}2,3 \\
4,1\end{array}$ \\
\hline $\begin{array}{l}\text { Humo-calcique colluvial } \\
\text { Hêtraie à adénostyle }\end{array}$ & $\begin{array}{l}A_{1} \\
A_{1} C\end{array}$ & $\begin{array}{c}0-20 \\
20-50\end{array}$ & $\begin{array}{l}75 \\
90\end{array}$ & $\begin{array}{l}59,5 \\
36,2\end{array}$ & $\begin{array}{l}30,5 \\
26,2\end{array}$ & $\begin{array}{l}8,3 \\
8,9\end{array}$ & $\begin{array}{l}1,1 \\
3,6\end{array}$ & $\begin{array}{r}0,6 \\
25,1\end{array}$ \\
\hline $\begin{array}{l}\text { Brun à pellicule calcaire } \\
\text { Hêtraie-sapinière mésophile }\end{array}$ & $\begin{array}{l}A_{1} \\
(B)\end{array}$ & $\begin{array}{c}0-15 \\
15-40\end{array}$ & $\begin{array}{r}0 \\
45\end{array}$ & $\begin{array}{l}33,7 \\
35,7\end{array}$ & $\begin{array}{l}41,5 \\
40\end{array}$ & $\begin{array}{l}21,6 \\
21,4\end{array}$ & $\begin{array}{l}2,7 \\
2,4\end{array}$ & $\begin{array}{l}0,5 \\
0,5\end{array}$ \\
\hline $\begin{array}{l}\text { Brun calcique } \\
\text { Hêtraie à dentaire }\end{array}$ & $\begin{array}{l}A_{1} \\
(B)\end{array}$ & $\begin{array}{c}0-15 \\
15-30\end{array}$ & $\begin{array}{l}15 \\
35\end{array}$ & $\begin{array}{l}45,8 \\
32\end{array}$ & $\begin{array}{l}28,8 \\
33,7\end{array}$ & $\begin{array}{l}14,6 \\
23,2\end{array}$ & $\begin{array}{l}7,4 \\
7,2\end{array}$ & $\begin{array}{l}3,4 \\
3,9\end{array}$ \\
\hline $\begin{array}{l}\text { Brun humifère de laizines } \\
\text { Hêtraie à érable mésotrophe }\end{array}$ & $\begin{array}{l}A_{1} \\
(B)\end{array}$ & $\begin{array}{c}0-25 \\
25-50\end{array}$ & $\begin{array}{r}0 \\
80\end{array}$ & $\begin{array}{l}43 \\
22\end{array}$ & $\begin{array}{l}32,7 \\
25,7\end{array}$ & $\begin{array}{l}11,7 \\
24,1\end{array}$ & $\begin{array}{r}8 \\
18\end{array}$ & $\begin{array}{r}4,6 \\
10,2\end{array}$ \\
\hline $\begin{array}{l}\text { Brun limoneux } \\
\text { Hêtraie-sapinière hygrophile }\end{array}$ & $\begin{array}{l}A_{1} \\
(B)\end{array}$ & $\begin{array}{c}0-15 \\
15-100\end{array}$ & $\begin{array}{l}0 \\
0\end{array}$ & $\begin{array}{l}27,8 \\
35,8\end{array}$ & $\begin{array}{l}47,6 \\
38,6\end{array}$ & $\begin{array}{l}20,5 \\
21,1\end{array}$ & $\begin{array}{l}3,7 \\
4\end{array}$ & $\begin{array}{l}0,4 \\
0,5\end{array}$ \\
\hline $\begin{array}{l}\text { Brun lessivé argileux } \\
\text { Hêtraie-sapinière hygrophile }\end{array}$ & $\begin{array}{l}A_{1} \\
A_{2} \\
(B)\end{array}$ & $\begin{array}{c}0-8 \\
8-25 \\
25-60\end{array}$ & $\begin{array}{l}0 \\
0 \\
0\end{array}$ & $\begin{array}{l}19,7 \\
23,5 \\
48,2\end{array}$ & $\begin{array}{l}37,2 \\
35,8 \\
23,8\end{array}$ & $\begin{array}{l}26,7 \\
24,2 \\
13,2\end{array}$ & $\begin{array}{l}16,2 \\
15,8 \\
14,1\end{array}$ & $\begin{array}{l}0,2 \\
0,7 \\
0,7\end{array}$ \\
\hline $\begin{array}{l}\text { Brun acide } \\
\text { Hêtraie à Millet }\end{array}$ & $\begin{array}{l}A_{1} \\
(B)\end{array}$ & $\begin{array}{c}0-10 \\
10-100\end{array}$ & $\begin{array}{l}10 \\
15\end{array}$ & $\begin{array}{l}16,9 \\
18,1\end{array}$ & $\begin{array}{l}21,2 \\
21,5\end{array}$ & $\begin{array}{l}15,9 \\
15\end{array}$ & $\begin{array}{l}26,8 \\
26,5\end{array}$ & $\begin{array}{l}19,2 \\
18,9\end{array}$ \\
\hline
\end{tabular}

contient $15 \%$ de cailloux calcaires, son $\mathrm{pH}$ est 6,6; l'horizon (B), de $15 \mathrm{~cm}$, est plus argileux et pius caillouteux ( $45 \%$ en poids). La base du profil est un horizon de $10 \mathrm{~cm}$ en farine calcaire argileuse. La réserve en eau estimée est meilleure que celle des sols humifères : $75 \mathrm{~mm}$ d'eau utile.

L'analyse factorielle des correspondance a regroupé avec les relevés de hê- traie à dentaire quelques relevés localisés sur des moraines au pied du Jura suisse. Ces sites montrent une flore plus acidicline : Galeopsis tetrahit, Galium rotundifolium, Milium effusum, Maianthemum bifolium. Les résidus granitiques ou gneissiques des moraines d'origine alpine permettent l'existence de sols bruns acides (Brunisol désaturé sur moraines acides). 
rassiennes et unité stationnelle correspondante (analyses effectuées au laboratoire d'analyse des

\begin{tabular}{|c|c|c|c|c|c|c|c|c|c|c|}
\hline $\begin{array}{c}\mathrm{CaCO}_{3} \\
(\%)\end{array}$ & $\begin{array}{l}\text { MO } \\
(\%)\end{array}$ & $\begin{array}{c}\text { Eau } 105^{\circ} \mathrm{C} \\
(\%)\end{array}$ & $\begin{array}{l}\text { C Réseau } \\
\text { sol }(\mathrm{mm})\end{array}$ & pHeau & $\mathrm{Ca}$ & $\underset{(m e q / 100 \mathrm{~g}}{M g}$ & g) $K$ & $\begin{array}{c}C \\
(\%)\end{array}$ & $\begin{array}{c}N \\
(\%)\end{array}$ & $C / N$ \\
\hline $\begin{array}{l}13,3 \\
29\end{array}$ & $\begin{array}{l}14,7 \\
11,1\end{array}$ & $\begin{array}{l}6,7 \\
5,4\end{array}$ & 57 & $\begin{array}{l}7,6 \\
7,9\end{array}$ & $\begin{array}{l}67,9 \\
61,9\end{array}$ & $\begin{array}{l}0,64 \\
0,52\end{array}$ & $\begin{array}{l}0,24 \\
0,17\end{array}$ & $\begin{array}{l}8,5 \\
6,43\end{array}$ & 0,64 & 13,2 \\
\hline $\begin{array}{r}4,1 \\
10,4\end{array}$ & $\begin{array}{l}9,9 \\
4,4\end{array}$ & $\begin{array}{l}5,5 \\
4,6\end{array}$ & 67 & $\begin{array}{l}7,7 \\
8,1\end{array}$ & $\begin{array}{l}59,2 \\
53,3\end{array}$ & $\begin{array}{l}0,57 \\
0,35\end{array}$ & $\begin{array}{l}0,27 \\
0,19\end{array}$ & $\begin{array}{l}5,78 \\
2,58\end{array}$ & 0,47 & 12,3 \\
\hline $\begin{array}{l}3,3 \\
4,3\end{array}$ & $\begin{array}{r}22,1 \\
8,6\end{array}$ & $\begin{array}{l}8,6 \\
6,4\end{array}$ & 60 & $\begin{array}{l}7,5 \\
7,9\end{array}$ & $\begin{array}{l}77,6 \\
60,5\end{array}$ & $\begin{array}{l}0,88 \\
0,63\end{array}$ & $\begin{array}{l}0,26 \\
0,21\end{array}$ & $\begin{array}{c}12,9 \\
4,98\end{array}$ & 0,81 & 15,9 \\
\hline $\begin{array}{r}2,7 \\
28,9\end{array}$ & $\begin{array}{r}20,7 \\
8,36\end{array}$ & $\begin{array}{l}8,3 \\
6,7\end{array}$ & 57 & $\begin{array}{l}7,5 \\
8\end{array}$ & $\begin{array}{l}75 \\
62\end{array}$ & $\begin{array}{l}1,19 \\
0,63\end{array}$ & $\begin{array}{l}0,42 \\
0,21\end{array}$ & $\begin{array}{l}12 \\
4,86\end{array}$ & $\begin{array}{l}0,85 \\
0,45\end{array}$ & $\begin{array}{l}14,1 \\
10,9\end{array}$ \\
\hline $\begin{array}{l}0 \\
0,7\end{array}$ & $\begin{array}{l}8,62 \\
3,65\end{array}$ & $\begin{array}{l}5,3 \\
4,1\end{array}$ & 88 & $\begin{array}{l}5,6 \\
7,4\end{array}$ & $\begin{array}{l}13,1 \\
27,4\end{array}$ & $\begin{array}{l}1,05 \\
0,29\end{array}$ & $\begin{array}{l}0,53 \\
0,2\end{array}$ & $\begin{array}{l}5,01 \\
2,12\end{array}$ & 0,36 & 14 \\
\hline $\begin{array}{l}0,5 \\
1,5\end{array}$ & $\begin{array}{c}12,6 \\
6,85\end{array}$ & $\begin{array}{l}6,2 \\
5,3\end{array}$ & 75 & $\begin{array}{l}6,6 \\
7,5\end{array}$ & $\begin{array}{l}37,7 \\
49,1\end{array}$ & $\begin{array}{l}1,18 \\
0,51\end{array}$ & $\begin{array}{l}0,29 \\
0,24\end{array}$ & $\begin{array}{l}7,31 \\
3,98\end{array}$ & 0,56 & 13 \\
\hline $\begin{array}{l}0,5 \\
4,3\end{array}$ & $\begin{array}{l}18 \\
11,1\end{array}$ & $\begin{array}{l}8,7 \\
7\end{array}$ & 75 & $\begin{array}{l}6,7 \\
7,7\end{array}$ & $\begin{array}{l}48,9 \\
63,2\end{array}$ & $\begin{array}{l}0,54 \\
0,32\end{array}$ & $\begin{array}{l}0,23 \\
0,19\end{array}$ & $\begin{array}{c}10,5 \\
6,44\end{array}$ & 0,79 & 13,2 \\
\hline $\begin{array}{l}0 \\
0\end{array}$ & $\begin{array}{c}10,4 \\
3,11\end{array}$ & $\begin{array}{l}5,1 \\
3,5\end{array}$ & 188 & $\begin{array}{l}5 \\
5,3\end{array}$ & $\begin{array}{l}9,4 \\
5,2\end{array}$ & $\begin{array}{l}1,33 \\
0,11\end{array}$ & $\begin{array}{l}0,44 \\
0,13\end{array}$ & $\begin{array}{l}6,07 \\
1,81\end{array}$ & 0,49 & 12,5 \\
\hline $\begin{array}{l}0 \\
0 \\
0\end{array}$ & $\begin{array}{r}5,6 \\
2,2 \\
0,95\end{array}$ & $\begin{array}{l}3,4 \\
1,8 \\
3,5\end{array}$ & 110 & $\begin{array}{l}4,7 \\
4,5 \\
5,2\end{array}$ & $\begin{array}{l}3,7 \\
0,7 \\
9,3\end{array}$ & $\begin{array}{l}0,36 \\
0,09 \\
0,46\end{array}$ & $\begin{array}{l}0,25 \\
0,11 \\
0,46\end{array}$ & $\begin{array}{l}3,25 \\
1,28 \\
0,55\end{array}$ & $\begin{array}{l}0,24 \\
0,08\end{array}$ & $\begin{array}{l}13,7 \\
16,2\end{array}$ \\
\hline $\begin{array}{l}0 \\
0\end{array}$ & $\begin{array}{l}4,28 \\
1,1\end{array}$ & $\begin{array}{l}1,7 \\
1,4\end{array}$ & 100 & $\begin{array}{l}4,8 \\
5\end{array}$ & $\begin{array}{l}2,4 \\
1,2\end{array}$ & $\begin{array}{l}0,32 \\
0,16\end{array}$ & $\begin{array}{l}0,11 \\
0,08\end{array}$ & $\begin{array}{l}2,49 \\
0,64\end{array}$ & 0,16 & 15,6 \\
\hline
\end{tabular}

La végétation qui recouvre ce type de sol est à rapprocher de la hêtraie à millet étalé ou Milio-Fagetum Frehner, 1963. Elle peut se rencontrer sur des placages de limons recouvrant le substrat calcaire, limons à l'origine de sols bruns lessivés (Rameau, 1988). De même, quelques relevés effectués sur des moraines alpines présentent des ressemblances avec la hêtraie à lu- zules (Luzulo-Fagetum Meus, 1937), qui se distingue des hêtraies calcicoles par $L u$ zula luzuloides, Luzula nivea, Veronica officinalis et Galium rotundifolium (Richard et Favarger, 1960). Leur faible nombre ne nous a pas permis d'individualiser ce groupe.

Le sol brun acide est épais (75 à plus de $110 \mathrm{~cm}$ ), acide ( $\mathrm{pH}$ eau $\left.A_{1}: 4,8\right)$ car il 




Fig 5. Altitude moyenne (petit trait horizontal) et amplitude de répartition des types de station selon l'altitude.

est constitué par un horizon (B) de $70 \mathrm{~cm}$ issu de dépôts morainiques acides de faible taille granulométrique : $45 \%$ de sables et $37 \%$ de limons dans la terre fine. Cette composition lui confère une bonne réserve en eau, environ $100 \mathrm{~mm}$ d'eau utile.

Nous avons vu au paragraphe Axe 2 de I'Interprétation de l'analyse factorielle des correspondances que l'axe 2 a été interprété comme un gradient de bilan hydrique croissant allant globalement de pair avec un gradient de $\mathrm{pH}$ décroissant. Dans le détail, ce parallélisme n'est pas toujours vérifié : les sols bruns calciques, peu acidifés, côtoient les sols bruns acides au centre du nuage de points projeté sur le plan $1 \times 2$ de l'AFC. Cependant ce regroupement de sites calcicoles avec des sites acidiphiles peut se comprendre en considérant la réserve en eau assez semblable de ces sols, proche de 75 à $100 \mathrm{~mm}$.

\section{Hêtraie à adénostyle}

Quelques relevés, très proches floristiquement de la hêtraie à tilleul, ont pu être rattachés à la hêtraie à adénostyle. L'Adenostylo-Fagetum Moor 1971, s'individualise grâce à quelques espèces neutrocalcicoles ou ne craignant pas les variations d'humidité du sol : Adenostyles alpina, Carex flacca, Centaurea montana, Hypericum hirsutum, Lathyrus vernus, Stachys officinalis... Le peuplement est habituellement dominé par le hêtre associé à l'érable sycomore. Le sapin et l'épicéa peuvent dominer aux altitudes moyennes et supé- 
rieures, ce qui indique que la hêtraie à adénostyle n'est pas une forêt pionnière. Cette association se rencontre entre 900 et $1100 \mathrm{~m}$, sur des éboulis grossiers, des bancs calcaires concassés, en position sommitale ou de mi-pente, sur des pentes $8-27^{\circ}$. Elle constitue un groupement vicariant altitudinal de la hêtraie à tilleul (Moor, 1971).

Les sols humifères sont le plus souvent du type humo-calcique colluvial (Calcisol humique caillouteux sur éboulis). L'horizon $A_{1}$ de $20 \mathrm{~cm}$ est constitué de $75 \%$ de cailloux calcaires de $2-20 \mathrm{~cm}$, la terre fine est très organique : $20-30 \%$ de matière organique; l'horizon brun $A_{1} C$ de $30 \mathrm{~cm}$ contient $90 \%$ de cailloux calcaires de moins de $10 \mathrm{~cm}$ de diamètre. La terre fine de l'horizon $A_{1}$, totalement décarbonatée, est constituée d'un complexe argilohumique à forte capacité d'échange cationique pouvant fixer plus de 70 meq de calcium pour $100 \mathrm{~g}$ de terre, ce qui est le maximum pour les sols échantillonés. Par contre, en raison de leur granulométrie, ces sols ont la plus faible réserve en eau potentielle : $60 \mathrm{~mm}$. Ce groupement se rencontre également sur des sols humocalcaires.

\section{Hêtraie-sapinière}

L'Abieti-Fagetum Oberd 1938 em 1957 est représenté dans notre échantillon par 53\% des relevés. Elle est caractérisée par Festuca altissima et Hordelymus europaeus. La CAH la subdivise en 2 groupes bien distincts : d'une part, les stations d'altitude moyenne, qui rassemblent les sousassociations "mésophile" et "hygrocline"; d'autre part un ensemble de stations sur des substrats plus drainants qui constituent la sous-association d'altitude ou «alticole». Les 2 premières sousassociations sont constituées de peuple- ments très sains; la défoliation moyenne est de $15 \%$ et le jaunissement est très peu fréquent. Les plantations réalisées sur la bordure ouest de massif appartiennent à ces groupements, de même que les plus belles forêts du massif : forêt de Levier, forêt de La Joux. La hêtraie-sapinière alticole est plus défoliée (19\%) et plus jaunissante que la moyenne des peuplements.

\section{Hêtraie-sapinière mésophile}

Toutes ses espèces les plus caractéristiques appartiennent préférentiellement à l'étage montagnard inférieur; elle partage ce dernier avec la hêtraie à laîches neutrophile en se développant dans des milieux nettement moins secs et moins calcaires, entre 710 et $880 \mathrm{~m}$. C'est pourquoi les espèces calcicoles, peu représentées, laissent la place aux espèces neutroclines à acidiclines : Arum maculatum, Athyrium filix-femina, Carex sylvatica, Circaea lutetiana, Dryopteris dilatata, Glechoma hederacea, Veronica montana. Cette forme de hêtraie-sapinière se rencontre surtout sur des plateaux en bancs calcaires diaclasés et parfois sur des éboulis sur pente faible $\left(4-19^{\circ}\right)$. Les précipitations sont de $1300-$ $1500 \mathrm{~mm}$ à ces altitudes.

Les sols sont des sols bruns de laizines (dans les fentes des lapiaz), bruns limoneux et surtout bruns à pellicule calcaire (Brunisol saturé, argilo-limoneux, mésosaturé en surface). Ce type de sol est constitué d'un horizon $A_{1}$ de $15 \mathrm{~cm}$ non calcaire, limono-argileux sans cailloux, à $\mathrm{pH} 5,6$ et d'un horizon (B) de $25 \mathrm{~cm}$ argilo-limoneux qui contient $45 \%$ de cailloux calcaires décomposés par l'eau sur leur surface en une pellicule calcaire d'altération. Malgré ce flux calcaire, le sol n'est pas saturé en calcium échangeable : 27 meq pour $100 \mathrm{~g}$. La réserve en eau totale est assez bonne : $90 \mathrm{~mm}$. Cette sapinière est la plus riche en sapins de tous les groupes; elle est exclu- 
sivement traitée en futaie régulière sous laquelle la végétation est moins abondante que sous les autres sapinières.

\section{Hêtraie-sapinière hygrocline}

Elle occupe les stations sur lesquelles le bilan hydrique est le plus favorable. La flore caractéristique comporte des espèces neutroclines, neutronitrophiles et surtout des espèces mésophiles ou hygroclines : Anemone nemorosa, Atrichum undulatum, Cardamine pratensis, $C$ flexuosa, Carex remota, $C$ pendula, Circaea intermedia, Impatiens noli-tangere, Lysimachia nemorum, Plagiomnium affine, $P$ punctatum, $P$ undulatum, Plagiochila asplenioides. Cette hêtraie-sapinière est étendue entre 790 et $1030 \mathrm{~m}$, sur des substrats assez imperméables : des marnes, des bancs calcaires compacts ou diaclasés situés au fond de vastes dépressions, sur des plateaux, en position de bas de pente ou sur pente faible $\left(3-14^{\circ}\right)$.

Les types de sols qui lui sont associés sont des sols bruns à pellicule calcaire ou des sols bruns eutrophes, les plus fréquents étant les sols bruns limoneux (Brunisol saturé, limoneux, pachique, mésosaturé en surface) et les sols bruns lessivés argileux (Brunisol désaturé, luvique, argileux).

Les sols bruns limoneux formés à partir de limons éoliens post-glaciaires (Pochon, 1978 ) sont les plus épais de tous, plus de $80-110 \mathrm{~cm}$, et ne contiennent pas de calcaire. Ils sont constitués d'un horizon $A_{1}$ de $15 \mathrm{~cm}$ à $\mathrm{pH} 5$ et d'un horizon $(B) \mathrm{com}$ portant $60 \%$ de limons et $30 \%$ d'argile. Ces caractéristiques leur confèrent la meilleure réserve en eau : $190 \mathrm{~mm}$.

Les sols bruns argileux comportent un horizon $A_{1}$ limoneux brun foncé de $8 \mathrm{~cm}$ avec peu de matière organique $(5,6 \%, \mathrm{pH}$ 4,5 ), un horizon $A_{2}$ de $20 \mathrm{~cm}$ brun-jaunâtre limono-argileux à pH 5,2 sans calcaire et un horizon (B) de 40 à $80 \mathrm{~cm}$ argileux, sans cailloux. Ces sols profonds, situés sur des stations de bas-de-pente ou des dépressions, ont une réserve en eau estimée de l'ordre de $110 \mathrm{~mm}$.

\section{Hêtraie-sapinière alticole}

Entre 900 et $1100 \mathrm{~m}$, la hêtraie-sapinière s'enrichit en espèces de l'étage montagnard supérieur et en espèces acidiclines : Asplenium viride, Dicranum scoparium, Festuca altissima, Hylocomium splendens, Moehringia muscosa, Orthilia secunda, Rhytidiadelphus triqueter, $R$ loreus, Ribes alpinum, Rosa pendulina, Rubus saxatilis, Vaccinium myrtillus. Ce groupement se localise sur les substrats perméables : bancs calcaires diaclasés ou concassés, éboulis fins ou grossiers, sur des pentes de 0 à $22^{\circ}$. Les précipitations s'élèvent à 1600-1900 mm et la température moyenne est $6-7^{\circ} \mathrm{C}$.

Deux variantes se distinguent en fonction du type de sol : la majorité des sols sont humifères (variante à sols humifères), particulièrement humo-calciques sur banc concassé (Calcisol humique, caillouteux, argileux, sur calcaire concassé); on peut également rencontrer des sols bruns à pellicule calcaire ou bruns de laizines (variante à sols bruns eutrophes superficiels). Les sols humocalciques sur banc concassé sont constitués d'un horizon $\mathrm{LA}_{0}$ de $5 \mathrm{~cm}$, organique, fibreux, brun-rougeâtre qui comporte $90 \%$ de cailloux calcaires anguleux; I'horizon $A_{1}$ de $20 \mathrm{~cm}$ gris très foncé est également riche en cailloux de faibles dimensions, la terre fine est très organique $(25-40 \%$ de matière organique), son $\mathrm{pH}$ est de 7,5; l'horizon $\mathrm{A}_{1} \mathrm{C}$ de $20 \mathrm{~cm}$ est un peu plus clair, plus argileux que son homologue du type «colluvial» mais également décarbonaté et riche en calcium (60-70 meq). Ce type de sol, rencontré sur des bancs calcaires purs, durs et très finement concassés, présente une mauvaise réserve en eau : $60 \mathrm{~mm}$. 
L'étage montagnard supérieur est le domaine de 2 associations dans lesquelles le sapin laisse progressivement la place à l'épicéa : la hêtraie à érable et la pessière à doradille. Les précipitations totales varient entre 1800 et 2600 , et la température moyenne est inférieure à $5^{\circ} \mathrm{C}$.

\section{Hêtraie à érable}

Cette association dominée par le hêtre et l'érable sycomore se rencontre entre 1100 et $1300 \mathrm{~m}$, en situations froides. Les espèces à tempérament montagnard caractérisent ce groupement de l'Aceri-Fagetum Bartsch 1940 : Festuca altissima, Moehringia muscosa, Polystichum aculeatum, Ranunculus aconitifolius, $R$ lanuginosus, Rosa pendulina, Rubus saxatilis, Saxifraga rotundifolia, Valeriana montana, Veronica urticifolia. Deux sous-associations se distinguent selon le caractère plus ou moins mésotrophe de la flore, en relation avec le type de sol.

\section{Hêtraie à érable neutrophile}

Sur les substrats drainants (bancs concassés, éboulis) à sols humifères sur pentes assez fortes $\left(5-32^{\circ}\right)$. Les espèces différentielles calcicoles, neutrocalcicoles ou neutroclines peuvent s'accommoder de sols assez secs : Corylus avellana, Festuca altissima, Lonicera alpigena, Moehringia muscosa, Sorbus aria, Valeriana montana, Veronica urticifolia. Cette sous-association se rencontre sur des sols assez semblables à ceux de la hêtraie-sapinière alticole mais, en plus de certaines différences floristiques, elle se localise sur des pentes plus prononcées et à des altitudes supérieures (200 $\mathrm{m}$ plus haut en moyenne). Cette dernière caractéristique est reflétée dans la flore par un enrichissement en espèces montagnardes : Acer pseudoplatanus, Adenostyles alpina, Lonicera alpigena, Saxifraga rotundifolia, Senecio fuschii,
Valeriana montana, Veronica urticifolia. Les sapins de cette unité ont montré les plus fortes défoliations $\langle 22,6 \%$ en moyenne) et le jaunissement est juste un peu inférieur à celui des hêtraies à laîches.

\section{Hêtraie à érable mésotrophe}

Sur les substrats moins drainants couverts par des sols habituellement plus désaturés : bruns à pellicule calcaire, bruns calciques ou bruns de laizines. Les espèces sont neutrophiles à acidiclines et certaines d'entre elles indiquent un bilan hydrique plus favorable que pour l'autre sousassociation : Athyrium filix-femina, Carex sylvatica, Fissidens taxifolius, Geum urbanum, Lysimachia nemorum, Plagiomnium undulatum, Paris quadrifolia, Plagiochila asplenioides, Primula elatior, Ranunculus lanuginosus. Quelques plantes de mégaphorbiaies, telles que Cicerbita alpina ou les renoncules peuvent s'y développer considérablement.

\section{Pessière à doradille}

Cette association de l'Asplenio-Piceetum Kuoch 1954, se rencontre sur les plateaux de haute altitude ( 1150 à $1310 \mathrm{~m}$ ) formant des lapiaz : bancs calcaires découpés en gros blocs. L'épicéa est davantage présent qu'au sein des autres groupements, ainsi que Asplenium viride (petite fougère : la doradille). Les espèces acidiclines ou acidiphiles différencient la station : Anthoxanthum odoratum, Dicranum scoparium, Maianthemum bifolium, Melampyrum sylvaticum, Polypodium vulgare, Polytrichum formosum, Rhytidiadelphus loreus, Vaccinium myrtillus.

Ce milieu constitue une mosaïque de sols : sol lithocalcique humifère de quelques $\mathrm{cm}$ sur les blocs et sol brun de laizines (Organosol Folist mésosaturé) dans les fentes (laizines). Le sol brun de laizines est constitué d'un humus de type mor sur 
un horizon $A_{11}$ de $8 \mathrm{~cm}$ brun foncé, riche en racines fines, myceliums et en matière organique (20\%), à pH 5; l'horizon $A_{12}$ de $15 \mathrm{~cm}$ est un peu plus compact et contient également $75 \%$ de blocs calcaires. L'horizon (B) brun jaunâtre de $25 \mathrm{~cm}$ est plus argileux et renferme plus de cailloux calcaires empilés entre lesquels descendent les grosses racines jusqu'à $50 \mathrm{~cm}$. La réserve en eau est estimée à $75 \mathrm{~mm}$. Le soutirage karstique intense, dû à des précipitations supérieures à $2000 \mathrm{~mm}$, appauvrit le complexe absorbant en calcium. En raison du faible effectif, les 2 variantes oligotrophe et mésotrophe qui peuvent être distinguées selon l'épaisseur de sol, ont été regroupées.

\section{Composition de la strate arborescente}

Tous groupements végétaux confondus, l'épicéa et le hêtre se rencontrent dans la strate arborescente de $75 \%$ des peuplements échantillonnés. L'érable sycomore est présent sur $24 \%$ des sites. Les autres espèces arborescentes sont nettement plus rares dans l'étage dominant : le frêne (fréquence : $11 \%$ ), le sorbier des oiseleurs $(5,3 \%)$, l'alisier blanc $(2,4 \%)$, le chêne sessile, le tilleul à grandes feuilles, l'érable plane (moins de $2 \%$ )...

En raison de l'autoécologie des essences et des interventions sylvicoles, la proportion de feuillus dans les peuplements de sapins change avec l'altitude; elle est maximale en dessous de $800 \mathrm{~m}$ et au-dessus de $1100 \mathrm{~m}$. Le caractère plus ou moins montagnard des espèces arborescentes, et sa prise en compte par des gestionnaires forestiers, conditionnent la composition des peuplements au long du gradient d'altitude : Abies alba, Fraxinus excelsior, Quercus petraea, Carpinus betulus, Acer campestre, A platanoides se raréfient ou disparaissent tandis que Picea abies, Fagus sylvatica, Acer pseudoplatanus, Sorbus aucuparia sont davantage présents aux altitudes élevées.

Les conditions stationnelles et sylvicoles modulent ces tendances générales et favorisent localement certaines espèces. Le sapin forme des peuplements presque purs au sein des hêtraiesapinières mésophiles et hygrophiles et des hêtraies à dentaire; ces groupes caractérisent les milieux les plus mésophiles et les altitudes moyennes (750-950 m). Le remplacement du sapin par l'épicéa quand l'altitude augmente est net dans la série hêtraie-sapinière alticole, hêtraie à érable, pessière à doradille. Au sein de l'échantillon, la régénération du sapin est plus abondante dans les groupes formés de peuplements clairs dans lesquels les sapins dominants sont en contact avec leurs voisins sur moins de $30 \%$ de la circonférence du houppier; c'est le cas de la hêtraie à laîches et de la pessière à doradille. L'enrichissement en sapin de groupements qui lui sont peu favorables créé des sylvofaciès au sein desquels les sapins montrent des jaunissements importants; c'est le cas en hêtraie à laîches et hêtraie à érable neutrophile.

Le caractère xérotolérant du hêtre explique sa plus grande abondance sur les stations sèches, particulièrement en hêtraie à tilleul et sa rareté en hêtraie-sapinière hygrophile. L'érable sycomore se rencontre essentiellement en hêtraie à érable et hêtraie à dentaire. On peut parfois trouver le frêne en hêtraie à laîches et hêtraie à tilleul.

\section{Phytogéographie}

L'interprétation du troisième axe de l'AFC a fait appel à la distribution des espèces au sein du massif jurassien, ce qui implique que la composition des groupements végétaux dépend de leur position géographi- 
que. Cette influence s'explique par la répartition hétérogène des conditions stationnelles en raison de la géomorphologie de la chaîne plissée calcaire. En effet, le relief du Jura est nettement dissymétrique : du côté français, une succession de plateaux entre 600 et $900 \mathrm{~m}$ d'altitude permet d'atteindre progressivement la Haute-Chaîne $(1400-1700 \mathrm{~m})$, tandis que le rebord oriental s'abaisse brutalement sur la plaine suisse $(500 \mathrm{~m})$. La moitié nord du massif est essentiellement constituée par les plateaux, ceux-ci se réduisent vers le sud pour laisser la place aux grands plis de la Haute-Chaîne. Cette double dissymétrie conditionne globalement la répartition des types de sols, des mésoclimats, des espèces végétales et, ainsi explique la séparation géographique de certains groupements floristiques (fig 1).

La partie septentrionale peut être divisée en 2 zones parallèlement à son axe. La hêtraie-sapinière (climax climatique) constitue de belles forêts sur les grands plateaux recouverts de limons de la moitié ouest; localement, selon le type de sol, on trouve la sous-association mésophile ou la sous-association hygrophile. La hêtraie à dentaire ou la hêtraie à tilleul se rencontrent sur le côté est, formé de plateaux calcaires. Les pentes fortes et les sols humifères carbonatés sont favorables à la hêtraie à tilleul; la hêtraie à dentaire se développe sur les sols bruns calciques des pentes faibles. La hêtraie à adénostyle se localise au sein de l'aire couverte par la hêtraie à dentaire, sur les stations froides en altitude, à forte pente.

Dans la moitié sud, la hêtraie à Carex alba forme des taches sur le bord sudouest du massif sur les stations xérocalcaricoles, où elle constitue un climax stationnel. Deux de nos sites représentent ce groupe sur le versant genevois, où il constitue un climax climatique grâce à l'effet de foehn du massif (Gillet et al, 1984). Deux climax climatiques se rencontrent dans la partie élevée du massif : la hêtraiesapinière d'altitude à l'étage montagnard moyen, disjointe des autres hêtraiesapinières, et la hêtraie à érable à l'étage montagnard supérieur. Les plateaux boisés des sommets de la Haute-Chaîne sont recouverts par la pessière à doradille; cette association constitue un climax stationnel sur certains sites à plus faibles altitude. Elle est principalement localisée sur une petite partie du Jura : massif du Risoux, Mont Tendre et Chasseron.

\section{DISCUSSION}

\section{Les unités stationnelles sont des «ensembles flous»}

Bien que l'échantillonnage utilisé pour cette étude n'ait pas été réalisé dans une optique purement typologique, les principaux groupes floristiques comportant des sapins en quantité assez importante ont pu être identifiés. Le choix du site de relevés a été effectué sans tenir compte du type de station auquel it serait rattaché après analyse. Cette démarche a eu l'avantage de recueillir une image du milieu proche de la réalité de terrain, ce qui a permis de retrouver le continuum écologique au sein des données floristiques et écologiques. Par contre, les limites entre les groupes de relevés n'apparaissent pas toujours très nettement lors des analyses. La typologie réalisée a donc été évaluée grâce à une méthode de classification par ensembles flous.

Avant l'analyse, les relevés ont été distribués dans les 11 groupes décrits plus haut; après analyse, chaque groupe contient encore des relevés lui appartenant préférentiellement, ce qui confirme la validité de son existence. Les groupes les 
plus solides ont conservé tous leurs relevés : hêtraie à laîche, hêtraie à tilleul, hêtraie à érable mésotrophe et pessière à doradille. Par contre, certains relevés ont été réattribués à un autre groupe issu de la classification hiérarchique; ces sites "hybrides" ont donc une composition floristique intermédiaire entre 2 types de stations. La quantité de relevés hybrides entre 2 groupes donne une indication sur leur degré de ressemblance écologique. Généralement, 2 unités stationnelles avec de nombreux relevés hybrides sont géographiquement voisines : une partie des relevés sont intermédiaires entre la hêtraie-sapinière mésophile et la hêtraiesapinière hygrophile, certains relevés de la hêtraie-sapinière alticole sont redistribués dans la hêtraie à laîches neutrophile ou dans la hêtraie à érable, qui la jouxtent à l'est et à l'ouest.

Les relevés les plus typiques d'un groupe lui appartiennent presque exclusivement; sur le plan des 2 premiers axes de l'AFC, ils forment un noyau concentré dans la partie de l'aire de variabilité écologique qui exprime le mieux le facteur responsable de lindividualisation du groupe. Par exemple, la hêtraie-sapinière hygrophile qui recouvre une large gamme d'altitudes $(640-1180 \mathrm{~m})$ et d'humidité du sol, est plus caractéristique entre 750 et 970 $\mathrm{m}$, sur les sols les plus humides (bruns limoneux ou bruns lessivés argileux). La mise en évidence des individus d'associations les plus caractéristiques permet de les utiliser pour affiner la description de l'ensemble floristique, du type de sol associé, de la fertilité des peuplements.

La grande variabilité stationnelle des hêtraies à dentaire ou des hêtraiesapinières laisse penser que, avec un échantillon plus important, certaines unités stationnelles pourraient être subdivisées jusqu'à définir de véritables «types de stations forestières" plus homogènes (Delpech et al, 1985).

\section{Comparaisons avec les études antérieures}

Les relevés supplémentaires choisis pour représenter les divers groupements se sont presque tous positionnés dans la partie du plan correspondant aux milieux relativement secs et plutôt en altitude. Globalement, l'ordre des groupes floristiques a été conservé au long des gradients d'altitude et d'humidité du sol. Par exemple, les 3 sous-associations de hêtraie-sapinière (melicetosum, typicum et adenostyletosum) décrites par Simmeray (1976) ont été bien classés des plus basses altitudes vers les plus hautes, mais leur position les place au sein de nos hêtraie-sapinières alticoles ou hêtraies à érable. En fait, cette étude avait concerné la région de SaintClaude et les relevés sont en majorité situés entre $1000 \mathrm{~m}$ et $1300 \mathrm{~m}$, ce qui explique l'absence des hêtraie-sapinière hygrophile et hêtraie-sapinière mésophile. Cela laisse également penser que notre hêtraie-sapinière alticole pourrait se subdiviser en une variante à sols humifères (Abieti-Fagetum melicetosum selon Simmeray) et une variante à sols bruns (Abieti-Fagetum typicum). La projection des relevés de hêtraie à laîches, de hêtraie à dentaire, de hêtraie à érable et de pessière à doradille de Richard (1961) ou de Gillet et al (1984) corrobore notre analyse de la végétation.

La classification ascendante hiérarchique a individualisé un groupe de 4 relevés qui ont été regroupés avec la hêtraiesapinière hygrophile en raison du faible effectif et des affinités floristiques. Ces sites ont une flore acidiphile qui se développe sur des sols bruns lessivés limoneux ou argileux; ces peuplements peuvent être rattachés à la sapinière à prêles (EquisetoAbietetum, Moor, 1952) qui se développe sur les stations les plus humides : fond de doline, de vallon, dépressions. La projec- 
tion de relevés anciens (Richard, 1961) coïncide bien avec la localisation de ce groupe sur le plan des 2 premiers axes de I'AFC.

\section{CONCLUSIONS}

L'analyse de la végétation des sapinières jurassiennes a mis en évidence les principaux facteurs écologiques qui conditionnent la composition floristique d'un site. La flore spontanée intègre de nombreuses caractéristiques du milieu, tant climatiques qu'édaphiques; par le jeu de la composition en espèces et de leur abondance, elle fournit des indications difficiles à obtenir par des mesures ou des descriptions stationnelles. L'importance de l'alimentation en eau a notamment été bien révélée par la flore alors que son évaluation pédologique nécessite des moyens plus lourds.

Les principales unités phytosociologiques, à partir du niveau "association", s'individualisent par la combinaison de l'altitude et du bilan hydrique du site, qui dépend de la position topographique, de la pente et du drainage. La notion opérationnelle de «type de station» ne correspond pas toujours à celle d'association, surtout dans le cadre des grandes associations climaciques au sein desquelles on peut distinguer de nombreuses sous-unités en fonction des nuances pédologiques et altitudinales.

Cette étude a distingué 11 principales unités stationnelles qui appartiennent à 6 associations : hêtraie à laîche, hêtraie à tilleul, hêtraie à dentaire, hêtraie-sapinière, hêtraie à érable et pessière à doradille. La grande étendue de la surface étudiée dans le massif du Jura a permis d'apprécier la variabilité écologique et de cerner la répartition géographique de chaque unité stationnelle. À travers l'analyse des divers types de groupements auxquels le sapin pectiné participe, et des conditions écologiques correspondantes, cette étude phytoécologique a précisé l'écologie du sapin sur toute l'étendue de son aire de répartition dans le Jura.

Dans la suite de nos travaux, cette typologie servira de base pour étudier les relations entre les conditions stationnelles et l'expression de dépérissements forestiers dans le Jura. En particulier, les peuplements de sapins de hêtraie à tilleul montrent des signes de dépérissement tandis que les peuplements géographiquement et floristiquement voisins de hêtraie à dentaire sont dans un état sanitaire plus satisfaisant. Les unités stationnelles seront également étudiées du point de vue de leur potentialité de croissance en hauteur et de l'évolution de la croissance radiale des arbres.

\section{REMERCIEMENTS}

Le travail présenté a constitué un des aspects de l'étude dendroécologique du dépérissement du sapin dans le Jura. Nous remercions la Direction de l'espace rural et des forêts, le programme Deforpa et l'INRA, qui ont financé ces recherches, $R$ Schipfer pour sa collaboration technique pendant de la phase de terrain, $M$ Becker et JL Dupouey pour leurs conseils lors des analyses, BS Tan pour son aide pédologique et 2 lecteurs anonymes pour leurs critiques et suggestions. Nous sommes reconnaissants envers les Services forestiers suisses des 4 cantons concernés par l'étude, envers l'Office national des forêts de Franche-Comté et de l'Ain pour leur autorisation de carottage et leurs indications sur les peuplements de sapins.

\section{RÉFÉRENCES}

Baize D, Girard MC (1990) Référentiel pédologique français. Troisième proposition. Ed AFES-INRA, Plaisir, $279 p$ 
Becker M (1982) Influence relative du climat et du sol sur les potentialités forestières en moyenne montagne. Exemple des sapinières à Fétuque (Festuca altissima Vill) dans les Vosges alsaciennes. Ann Sci For 39, 1-32

Becker M (1987) Bilan de santé actuel et rétrospectif du sapin dans les Vosges. Étude écologique et dendrochronologique. Ann Sci For 44, 379-402

Becker M, Lévy G (1988) A propos du dépérissement des forêts : climat, sylviculture et vitalité de la sapinière vosgienne. Rev For Fr 40, 345-358

Bert GD (1992) Potentialité du sapin pectiné (Abies alba Mill) et qualité du bois sur les stations de sapinière dans le Jura (France et Suisse). Rev For Fr (sous presse)

Bert GD, Becker M (1990) Vitalité actuelle et passée du sapin (Abies alba Mill) dans le Jura. Étude dendroécologique. Ann Sci For 47, 395-412

Bruckert S, Gaiffe M (1980) Analyse des facteurs de formation et de distribution des sols en pays calcaire ou karstique. Ann Sci Univ Besançon, $4^{\theta}$ Sér Biol Vég, 19-67

CPCS (1967) Commission de pédologie et de cartographie de sols : classification des sols. ENSA, Grignon, $96 \mathrm{p}$

Delpech R, Dumé G, Galmiche P (1985) Typologie des stations forestières. Vocabulaire. IDF, Paris, $243 p$

Dupouey JL (1985) Intérêt de la notion d'ensemble flou en phytosociologie forestière. Application à la classification des relevés de végétation. In: Colloques Phytosiologiques. 14, "Phytosociologie et foresterie", 43-53

Dupouey JL (1989) Classification floue non hiérarchique : le programme Fuzzy. Rev Modulad 4, 1-7

Equihua M (1990) Fuzzy clustering of ecological data. J Ecol 78, 519-534

Gaiffe M, Schmitt A (1980) Sols et végétation à l'étage montagnard dans les forêts du Jura Central. Science du sol. Bull AFES 4, 265296

Gillet F, Lhote P, Trivaudey MJ (1984) Étude cartographique de la végétation et des milieux naturels du Jura Gessien. Laboratoire de Taxonomie expérimentale et de Phytoso- ciologie. Doc Univ Besançon, $82 \mathrm{p}+$ annexes

Guinochet M (1973) Carte phytosociologique Pontarlier 5-6 au 1/20 000. In: Phytosociologie. Masson, Paris, $227 \mathrm{p}$

Halliday G, Beadle M (1983) Flora Europaea. 5 Vol. Cambridge Univ Press

Lebart L, Morineau A, Tabard N (1977) Techniques de la description statistique, méthodes et logiciels pour l'analyse des grands tableaux. Dunod, Paris

Lebart L, Morineau A, Lambert T (1988) SPAD $N$ : système portable l'analyse des données. Manuel de réference. Version 1.2. CISIA, Sèvres

Moor M (1952) Die Fagion-Gesellschaften (Buchen, Tannen-Buchen und Ahornwälder) im Schweizer Jura. Beiträge zur geobotanischen Landesaufnahme der Schweiz Heft 31. Hans Huber, Berne, $200 \mathrm{p}$

Moor M (1968) Der Linden-Buchenwald. Vegetatio. Acta Geobot Vol 16, Fasc 1-4, 159191

Moor M (1971) Adenostylo-Fagetum Höhenvikariant des Linden-Buchenwaldes. Bauhinia. 4

Moor M (1972) Versuch einer soziologischsystematischen Gliederung des CariciFagetum. Vegetatio 24, 1-3, 31-69

Pfister RD (1989) Basic concepts of using vegetation to build a site classification system. In: Proceedings-Land classifications based on vegetation: applications for resource management. Intermountain Research station, Odgen, UT, 22-29

Pochon M (1978) Origine et évolution des sols du Haut-Jura suisse. Thèse de l'Université de Neuchâtel, $190 \mathrm{p}$

Rameau JC (1987) Contribution phytoécologique et dynamique à l'étude des écosystèmes forestiers : application aux forêts du Nord-Est de la France. Thèse Université Besançon. $340 \mathrm{p}$

Rameau JC (1988) Les Hêtraies mésoneutrophiles et acidiclines (Milio-Fagetum) du NordEst de la France. Doc Phytosoc. Nouvelle série. Vol 11. Camerino. Univ degli Studi, 205-220

Rameau JC, Schmitt A, Bidault M, Gaiffe M (1980) Végétation et écologie des forêts 
comtoises. In: Bull Soc Hist Nat Doubs Assoc UNIVERS. $\mathrm{N}^{\circ}$ spécial. 80-116

Richard JL (1961) Les forêts acidophiles du Jura. Étude phytosociologique et écologique. Matériaux pour le levé géobotanique de la Suisse. Fascicule 38, Hans Huber Berne. $164 \mathrm{p}$

Richard JL, Favarger C (1960) Les enclaves de végétation acidophile dans le Jura et le pro- blème du climax. In : Rapports du sol et de la végétation. Masson, Paris, 99-109

Saporta G (1990) Probabilités, analyses de données et statistiques. Technip, Paris

Simmeray J (1976) Essai d'interprétation des groupements végétaux de la région de SaintClaude en vue d'une synthèse cartographique. Thèse Univ Besançon. $214 p$ 